Math. Struct. in Comp. Science (2001), vol. 11, pp. 299-300. Printed in the United Kingdom

(c) 2001 Cambridge University Press

\title{
Preface to Locus Solum
}

The theory presented here is a new, radical step in the research program that started with linear logic and aiming at an interactive, resource and space conscious account of reasoning and programming. In the view of the author of this preface, some of the main innovations underlying ludics are:

- The explicit focus on interactivity in the definition of the logical connectives. The basic objects of the theory, called designs, are sorts of (untyped) Böhm trees, that are evaluated in a context (itself expressed as a set of designs) through a very simple interaction algorithm (the normalization of designs). Several related recent finite or even not so recent, works in some way or another, have identified similar objects, called sequential algorithms, strategies, or evaluation trees, etc. But in ludics, the formulas are then defined as behaviours, which can be seen as collections of tests: so all the logic is a derivation of the initial concept of interactivity. Jean-Yves Girard had to reject many attempts that were not interactive before stabilizing his work in the form presented here.

- Instrumental in the emergence of the simple and elegant notion of design was the concept of focalisation, due to Jean-Marc Andreoli, but having its roots in Girard's early recognition that (forgetting about exponentials) the connectives were split not only as additive and multiplicative connectives, but also into positive/irreversible and negative/reversible ones. Respecting this splitting, and grouping together successive occurrences of connectives of the same polarity, simplifies proof search considerably this was Andreoli's motivation. But also, the induced change of granularity permits a simple, alternating account of interactivity: the proof plays, then the counter-proof plays, then the proof plays, etc. It permits such theorems as the separation theorem: two designs are the same if and only if they are not distinguishable by any test.

- The focus on allocation consciousness: computing agents may compete to get resources in limited number (linear logic), but may also compete in accessing or storing resources: allocation issues become part of the logic. This has a number of consequences in the definition of logical connectives. The additive conjunction becomes a particular delocated case of an intersection connective, the latter giving a logical status to intersection types and subtyping. There is not a single tensor, but several, according to different protocols of interference on shared locations. The quantifiers enjoy unusual commutations.

- The introduction of the concept of paraproof, or proof that stops short somewhere, the interactive interpretation being 'give up' (the task of justifying the proof further). The author of this preface views this on the programming side as an emission and propagation of errors. If I want to test that a program for addition is one that is defined by recursion on the left argument first, I let it interact with a test that is just feeding the program with two arguments, the first being an error, the second a diverging program. Then the program passes the test if and only if it is defined by recursion on the left argument first. Just as in this example, but on the logical side, 
paraproofs fill the space and provide enough tests. Errors have been dealt with in a few theoretical works on the semantics of programming languages, but no logical account for them has been given previously.

In addition to being a thought-provoking fundamental paper, Locus Solum is also an exercise in scientific criticism. The paper itself is followed by a long dictionary, which serves both as a glossary for important concepts and as a historical and critical index. The style is free, ironic at times, largely humorous. Comedy is often cruel, but is generally considered healthy. In any case, I plead that scientific writing should be considered a part of writing. Parables and jokes may help in understanding concepts, in rejecting some dead ends, or in identifying controversial issues. In short, style counts in science. Good reading!

Pierre-Louis Curien March 2001 